Recepción: 31 / 10 / 2016

Aceptación: 11 / 01 / 2017

Publicación: 29 / 04 / 2017

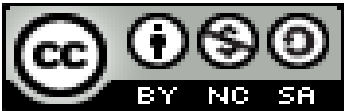

Ciencias económicas y empresariales

Artículo de investigación

\title{
Una mirada acerca de la enseñanza y aprendizaje del idioma inglés
}

\author{
A look at teaching and learning the English language
}

\section{Um olhar sobre o ensino e aprendizagem de Inglês}

Luís A. Veloz-Andrade ${ }^{\mathrm{I}}$

lveloz@espoch.edu.ec

Washington G. Mancero-Orozco ${ }^{\text {II }}$ wmancero@espoch.edu.ec

Correspondencia: 1veloz@espoch.edu.ec

\footnotetext{
I Magister en Administración para el Desarrollo Educativo, Ingeniero Agrónomo, Licenciado en Ciencias de la Educación Profesor de Enseñanza Media en la Especialización de Idiomas: inglés y francés, Docente de la Escuela Politécnica de Chimborazo ESPOCH, Riobamba, Ecuador.
}

" Magister en Administración para el Desarrollo Educativo, Licenciado en Ciencias de la Educación Profesor de Enseñanza Media en la Especialización de Idiomas: inglés y francés, Docente de la Escuela Politécnica de Chimborazo ESPOCH, Riobamba, Ecuador. 


\title{
Resumen
}

En la actualidad, en la mayoría de los campos laborales, existe una creciente demanda de profesionales con un alto dominio del idioma inglés, lo cual implica un mayor compromiso por parte de las instituciones educativas y sus actores, para formar ciudadanos competitivos en este aspecto. Con la presente revisión bibliográfica, se aportan elementos acerca del proceso de enseñanza y aprendizaje del idioma inglés y brinda algunos resultados de investigaciones sobre el tema.

Palabras clave: enseñanza; educación; lenguaje.

\begin{abstract}
At present, in most of the labor fields, there is an increasing demand for professionals with a high command of the English language, which implies a greater commitment on the part of the educational institutions and their actors, to form competitive citizens in this aspect. With the present bibliographic review, elements are provided about the teaching and learning process of the English language and provides some research results on the subject.
\end{abstract}

Keywords: teaching; education; language.

\section{Resumo}

Atualmente, na maioria dos campos industriais, há uma crescente demanda por profissionais com alta proficiência em Inglês, o que implica um maior compromisso das instituições e atores educacionais a serem cidadãos competitivos a este respeito. Com esta revisão da literatura, elementos sobre o processo de ensino e aprendizagem de Inglês são fornecidos e fornece alguns resultados de pesquisa sobre $\mathrm{o}$ assunto.

Palavras chave: educação; Educação; linguagem.

\section{Introducción}

La entrada al nuevo milenio, los avances científicos y tecnológicos, así como el desarrollo histórico-social, han condicionado el significativo progreso del mundo en el que están involucrados los profesionales de las diferentes esferas. (González Ortiz, L, Álvarez López, M E, Reyna González, M. 2015). 
Todo lo anterior ocurre en un sistema globalizante, que obliga a comunicarse en un segundo idioma por lo general el inglés y, por ende, a trazar estrategias de comunicación entre las personas en todo el universo, proporcionando así tácticas encaminadas a resolver los problemas que emergen en el propio contexto comunicativo. (González Ortiz, L, Álvarez López, M E, Reyna González, M. 2015).

$\mathrm{Al}$ respecto, la formación de estudiantes en la actualidad, demanda del conocimiento del inglés como herramienta que le va a permitir profundizar, desde la ciencia en los avances que a diario se publican en este idioma. De igual manera, en el intercambio que tiene en el marco de foros, congresos y diferentes eventos, emerge la necesidad de usar el inglés para lograr la comunicación oral entre hablantes de otros idiomas que se valen de este para lograr el entendimiento. Lo anterior justifica el que en la actualidad se enseñe y se aprenda inglés con fines profesionales en el área educativa. (González Ortiz, L, Álvarez López, M E, Reyna González, M. 2015).

El objetivo de este trabajo tiene como punto de partida algunos aspectos que concierne al proceso de enseñanza y aprendizaje del idioma inglés.

\section{Desarrollo}

Las diferencias entre individuos, sus condiciones de aprendizaje y los contextos en los que aprenden son algunos aspectos sobre los cuales se focalizan los investigadores para deducir y demostrar la complejidad del aprendizaje de una lengua extranjera. (Manga M. A. 2008).

Manga M A (2008) expresa que los procesos de aprendizaje de la lengua extranjera constituyen un interés de suma consideración para los que se mueven en el campo de la docencia. Desde esta óptica, Santos Gargallo (1999: 22) destaca que:

"A todos los profesores nos interesa profundizar en el conocimiento del proceso de aprendizaje, es decir, en los factores psico-afectivos, sociales, educativos, etc. que inciden en dicho proceso; ya que cuánto más sepamos sobre este proceso, mejor podremos orientar el de enseñanza, en el sentido de que contribuya a agilizar y facilitar el uso de la nueva lengua.

El aprendizaje de una lengua extranjera debe siempre tener en cuenta varios factores que lo facilitan o lo dificultan según las situaciones. Muchos estudios han sido realizados sobre dichos factores y permiten una mejor comprensión del concepto de aprendizaje de la lengua extranjera 
por los profesionales de la docencia. En este sentido, cabe resaltar el estudio de M. Bernaus (2001: 80) en el que aparecen algunos de estos factores "agrupados en tres categorías: a) factores biológicos y psicológicos: edad y personalidad; b) factores cognitivos: inteligencia, aptitudes lingüísticas, estilos y estrategias de aprendizaje, y c) factores afectivos: actitudes y motivación. (Manga M. A. 2008).

En este orden de ideas se puede citar, que cada ser humano tiene características particulares y es diferente de los demás. Estos rasgos le afectan positiva o negativamente y tienen implicaciones muy notables en el aprendizaje de una lengua extranjera. La personalidad reúne algunas dimensiones del comportamiento que adopta el alumno al estudiar una lengua extranjera. El aula pasa a ser un contexto de encuentro de varias dimensiones de la personalidad de los alumnos que el docente debe gestionar. (Manga M. A. 2008).

En la actualidad, el inglés es uno de los idiomas más hablados en el mundo (...) se ha convertido en la lengua franca, por lo que constituye el principal idioma de comunicación internacional Además, en el ámbito internacional, hoy más que nunca resulta imprescindible aprender este idioma, pues su uso se extiende a casi todas las áreas del conocimiento y desarrollo humano. En la era de la globalización el inglés ha sido considerado como la lengua internacional, una lengua franca que afecta, en mayor o menor medida, a los diversos campos disciplinarios y profesiones. En otras palabras, es una necesidad evidente, pues se trata de la herramienta que permite la comunicación con personas e instituciones de otros países, y la forma en que se enseña adquiere un carácter trascendental, pues son precisamente las formas de enseñar o las estrategias implementadas en la enseñanza, las que darán como resultado el éxito o el fracaso en el logro de la competencia comunicativa. (Mayoral-Valdivia, P. J. 2016).

Los docentes de inglés de los colegios, están en constante capacitación a fin de mejorar el proceso de enseñanza aprendizaje, el resto de instituciones educativas particulares buscan las mejores estrategias metodológicas, materiales exclusivos, personal en pocos casos nativos de habla inglesa, para impartir las clases de inglés dando mayor relevancia y énfasis en la importancia de aprender este idioma. (Mayoral-Valdivia, P. J. 2016).

El aprendizaje de esta lengua, no solo se limita a hablar la misma, sino a conocer a su gente, costumbres, cultura e historia de sus pueblos y de todo el mundo. La visión de cada centro 
educativo es formar jóvenes de manera competentes para enfrentar las dificultades y necesidades que el mundo actual exige en el desarrollo intelectual en su vida estudiantil y más tarde profesionalmente.

Algunas reflexiones del autor sobre la Enseñanza y aprendizaje del idioma ingles Después de una revisión de la literatura referente al tema el autor reflexiona que el proceso enseñanzaaprendizaje es una tarea muy compleja cuando no se cuenta con las herramientas adecuadas, muchos han sido los investigadores que desde tiempos muy lejanos han venido buscando las estrategias más adecuadas para facilitar este proceso. Aprender un nuevo idioma implica dificultad en cada una de las destreza que hay que desarrollar como son la destreza de escuchar, al estudiante se le dificultan los sonidos de las palabras porque los fonemas no son los mismos que en español, al leer, no comprende las palabras en la parte de la estructura de la oración, los adjetivos van antes de los sustantivos, los verbos algunas veces son sustantivos o viceversa. En la destreza de hablar la dificultad están en que pronuncian los sonidos como los escriben, limitando su comunicación por temor a equivocarse o que se rían de cómo pronuncian. En la destreza de escribir la complicación es que la mayoría de las palabras, no se escriben como se pronuncian, creándose una apatía al estudio del nuevo idioma.

Al mismo tiempo, considera que todos estos antecedentes hacen que los estudiantes se bloqueen en el aprendizaje del inglés, por lo que se pueden observar manifestaciones en los estudiantes como: escaso conocimiento del idioma inglés que se evidencia al dirigirles una pequeña frase que no la saben comprender ni responder, la falta de necesidad de aprender el idioma inglés debido a que en el medio en el que viven no lo requieren y además se percibe la apatía que tienen los estudiantes a la utilización del nuevo idioma en la comunicación oral diaria cuando este debería ser el laboratorio para un mejor aprendizaje, la influencia de un lenguaje de claves y símbolos muy común entre jóvenes, usado en internet, en el chat y en la comunicación a través del celular.

Es evidente que se debe de tener en cuenta algunos aspectos importantes en el aprendizaje del idioma inglés como la motivación, la utilización del material pedagógico por parte del maestro, las estrategias metodológicas que se emplean en el desarrollo didáctico. .

No hay un estilo puro o único, la necesidad de cada persona es diferente por lo tanto el interés en el aprendizaje también es diferente, los estilos son varios pero siempre hay uno predominante. 
Las estrategias están ligadas al estilo de aprendizaje que es usado, por esta razón cada estilo necesita de una estrategia diferente. Desde esta óptica, la relevancia que tiene investigar las estrategias didácticas para la enseñanza de inglés se hace evidente.

- Investigador como Mayoral-Valdivia, P. J. (2016) en la literatura revisada aprecia una gran variedad de estilos, enfoques y formas de abordar el tema de la enseñanza de lenguas como: Estudios basados en la literatura y centrados en habilidades lectoras,

- Investigación sobre modelos y enfoques de enseñanza centrados en el estudiante,

- Estudios enfocados al desarrollo de competencias,

- Documentos enfocados en la formación docente,

- Estudios fundamentados en teorías de la adquisición de lenguas,

- Estudios centrados en la edad del niño y manuales de estrategias,

- Investigaciones sobre el juego como estrategia de enseñanza-aprendizaje,

- Documentos sobre metodologías de investigación y métodos de enseñanza,

- Investigaciones sobre las teorías del desarrollo y tecnologías en la enseñanza de lenguas,

- Estudios sobre temas poco comunes relacionados con la enseñanza de lenguas. La gran diversidad muestra los temas en los que se centra la investigación y por consiguiente la falta de conocimiento.

\section{Impacto de métodos de la didáctica en el proceso enseñanza y aprendizaje del idioma inglés}

Los procesos de enseñanza y aprendizaje se enfrentan a una sociedad basada en el conocimiento, y desde la perspectiva de los educadores, es la sociedad del aprendizaje, donde el papel protagónico es para la innovación, la educación, la experiencia basada en conocimientos tácitos y la creatividad de los individuos, en la cual son necesarias. (Navas-Montes, Real-Poveda, Pacheco-Mendoza, Mayorga-Albán 2015).

En los últimos cinco años ha habido un considerable número de estudios sobre la enseñanza de inglés, en temas diversos (Kao, 2015; Adler, 2015; Davis \& Degotardi, 2015; Yangin \& Eksik, 2015; Ribeiro, 2015; Quig \& Chang Ha, 2015; Cummins \& Persad, 2014; entre otros). Estos estudios no abordan de manera específica las estrategias para la enseñanza de inglés y tampoco consideran la población analizada en este estudio. Uno de los pocos casos que abordan el tema es el de Kao (2015). Este autor presenta el uso de narrativa, en la región Asia-Pacífico, con el 
objetivo de facilitar que los niños transiten de un estado de simple escucha a uno activo en el que el niño se convierta en narrador de historias. Si bien el estudio es aplicado en un campo similar, las estrategias utilizadas giran en torno a la narrativa y es más una propuesta de enseñanza que un estudio desde las voces de los actores. (Mayoral-Valdivia, P. J. 2016).

Otros estudios se enfocan en la capacitación de los maestros de lenguas como el de Adler, (2015) y el caso de Davis, Degotardi (2015). Ambos observan la práctica docente para fomentar el trabajo en pares y observar el efecto que tiene en el aprendizaje de inglés en niños. Si bien hay similitudes con el presente estudio, ambos documentos ven el trabajo en pares como foco y no las estrategias de enseñanza.

Los autores Navas-Montes, Real-Poveda, Pacheco-Mendoza, Mayorga-Albán (2015) consideran los Entornos Virtuales de Aprendizaje (EVA), como instrumentos de mediación en los procesos de enseñanza y aprendizaje del idioma Inglés y a través de ellos se ofrece una oportunidad de perfeccionar la praxis académica, con el establecimiento de una estrategia que guíe dicho proceso, enmarcada en el aprendizaje productivo y cooperativo. Parten de la aseveración que la educación cada día tiende hacia el uso de las tecnologías apoyándose en el uso del computador, la telemática, múltiples tipos de redes, multimedia, hipertextos, realidad virtual, entre otros, por lo cual, los educadores están tendiendo a la utilización de EVA, que facilitan los procesos de enseñanza y aprendizaje, lo que genera ambientes más atrayentes al usuario, envolventes y multisensoriales.

Según Hernández Chérrez (2014), en su tesis doctoral el "modelo b-learning" que se planteó, no solo produjo un impacto positivo en el aspecto emocional de los alumnos, sino también en sus competencias y habilidades comunicativas en el idioma inglés. Con el "modelo b-learning" se beneficiaron las posibilidades y ventajas del aprendizaje virtual para propiciar aprendizajes significativos, desarrollar formas más dinámicas y flexibles para la comunicación, obtener y compartir información, así como trabajar de modo cooperativo.

En una investigación realizada por Jiménez Redruejo (2014), acerca de aprendizaje de inglés en alumnos de educación primaria a través del teatro, valido la capacidad de este recurso para motivar al alumnado a los cuales solían mantenerse al margen de la clase sin participación. Por otro lado la valoración del alumnado de modo holístico, ya que todos se sintieron integrados en el 
mismo proyecto, independientemente de sus capacidades individuales lo cual posibilitó un escenario con inteligencias múltiples donde todos los niños se sintieron útiles.

Otros investigadores Díaz L C; Martínez I P.; Roa G I., Sanhueza M G. (2010) estudiaron las cogniciones pedagógicas de diez jóvenes estudiantes de pedagogía en inglés, en su último año de formación en la universidad y que realizan su práctica profesional en establecimientos educativos chilenos. Los resultados revelan, que los informantes muestran en sus discursos una fuerte orientación hacia un proceso de enseñanza y aprendizaje del inglés, centrado en un modelo didáctico ecléctico de la lengua.

Por su parte Carvajal-Portuguez, Zayra Elisa (2013), desarrolla una propuesta innovadora para la enseñanza del inglés en secundaria, específicamente en el currículum operativo; es decir, el currículum que se lleva a cabo entre el docente y sus estudiantes en el aula. La propuesta considera instrumentos necesarios en la enseñanza del idioma inglés, entre ellos se cuenta con el instrumento de valoración y los indicadores para determinar el nivel del estudiantado, así como planeamientos de actividades para ser utilizadas en las distintas destrezas de este idioma. Es relevante indicar que los instrumentos exponen cuatro destrezas del idioma inglés: escuchar, leer, hablar y escribir; tal y como se plantea en el Programa de Estudio de la Enseñanza del Inglés Educación Diversificada del Ministerio de Educación Pública de Costa Rica.

En el programa de la disciplina Idioma Inglés, la disciplina Idioma Inglés forma parte de las disciplinas de Formación General de la carrera de Medicina en los Centros de Educación Médica Superior en Cuba. Estudios realizado por Montané González, B, Trujillo Chong, O E, Cruz Gorgoy, H, González Barrera, J M. (2014), reveló el tratamiento metodológico de la habilidad expresión oral muestra insuficiencias en el conocimiento de su didáctica y en la enseñanza basada en tareas expresadas en no aplicar métodos que garanticen la construcción de conocimientos.

Finalmente, resulta evidente, que aún con la diversidad conceptual que caracteriza el tratamiento teórico del inglés para la enseñanza-aprendizaje en un determinado contexto profesional, es importante conocer, analizar y comprender a mayor profundidad las características de las estrategias, empleadas en la práctica, para la enseñanza del idioma inglés. 


\section{Referencias bibliográficas}

Adler, M. A. (2015). Early childhood challenge: preparing high-quality teachers for a changing society in the USA. In L. Huo, \& D. 10.1007/978-3-662-44986-8_7 (Ed.), Early childhood education in three cultures. Berlin, Alemania: New Frontiers of Education Research.

Carvajal-Portuguez, Zayra Elisa (2013). Enseñanza del inglés en secundaria: una propuesta innovadora Educación, 37(2), pp. 79-101 Universidad de Costa Rica San Pedro, Montes de Oca, Costa Rica

Davis, B., \& Degotardi, S. (2015). Educator's understanding of, and support for, infant peer relationships in early childhood settings. (D. 10.1177/1476718X14538600, Ed.) Journal of Early Childhood Research, 13 (1), 64-78.

Díaz L C; Martínez I P.; Roa G I., Sanhueza M G.(2010). La enseñanza y aprendizaje del inglés en el aula: una mirada a las cogniciones pedagógicas de un grupo de jóvenes estudiantes de pedagogía1. (Consultado el 5 de febrero de 2016). Recuperado de www.scielo.org.ve/scielo.php?script=sci_arttext\&pid=S1316

González Ortiz, Lorena, Álvarez López, María Elena, \& Reyna González, María de los Ángeles. (2015). Análisis epistemológico de la comunicación oral del inglés con fines profesionales. MEDISAN, 19(3), 444-450. (Consultado el 1 de abril de 2016). Recuperado de http://scielo.sld.cu/scielo.php?script=sci_arttext\&pid=S1029-

$30192015000300019 \& \operatorname{lng}=\mathrm{es} \& \operatorname{lng}=\mathrm{es}$.

Hernández Chérrez (2014). El B-learning como estrategia metodológica para mejorar el proceso de enseñanza-aprendizaje de los estudiantes de inglés de la modalidad semipresencial del departamento especializado de idiomas de la Universidad Técnica de Ambato. (Tesis doctoral). (Consultado el 5 de abril de 2016). Recuperado de eprints.ucm.es/29610/1/T35913.pdf Jiménez Redruejo (2014). Aprendizaje de inglés en alumnos de educación primaria a través del teatro. (Consultado el 5 de abril de 2016). Recuperado de https://biblioteca.unirioja.es/tfe_e/TFE000740.pdf 
Mayoral-Valdivia, P. J. (2016). Estrategias didácticas para la enseñanza del idioma inglés a niños de preescolar: el caso de un colegio en Colima, México. Tesis de doctorado, Doctorado Interinstitucional en Educación. Tlaquepaque, Jalisco: ITESO. (Consultado el 7 de abril de 2016). Recuperado de http://quijote.biblio.iteso.mx/licencias/CC-BY-NC-ND-2.5-MX.pdf

Manga M A (2008). Enseñanza y aprendizaje de una lengua extranjera. Recuperado de https://www.um.es/tonosdigital/znum16/secciones/estudios--10-Ensenanza.htm

Montané González, Bárbara, Trujillo Chong, Oscar Eleuterio, Cruz Gorgoy, Hanny, \& González Barrera, Jesús María. (2014). El proceso enseñanza aprendizaje del ciclo de Inglés General: su estado actual. Revista de Ciencias Médicas de Pinar del Río, 18(4), 607-620. (Consultado el 7 de abril de 2016). Recuperado de http://scielo.sld.cu/scielo.php?script=sci_arttext\&pid=S1561$31942014000400007 \& \operatorname{lng}=$ es\&tlng=es.

Navas-Montes, Real-Poveda, Pacheco-Mendoza, Mayorga-Albán (2015). Los Procesos de Enseñanza y Aprendizaje del Idioma Inglés a través de los Entornos Virtuales de Aprendizaje. Revista Ciencia UNEMI 8 (13), pp. 47 - 55 